\title{
Blink Test Enhances Ability to Screen for Dry Eye Disease
}

\author{
James S Wolffsohn BSc MBA PhD ${ }^{1,2}$ \\ Jennifer P Craig BSc MSc PhD ${ }^{1,2}$ \\ Maria Vidal-Rohr BSc ${ }^{1}$ \\ Sonia Trave Huarte $\mathrm{BSc}^{1}$ \\ Lexia Ah-Kit BOptom² \\ Michael Wang MBChB ${ }^{2}$
}

1 Ophthalmic Research Group, Life and Health Sciences, Aston University, Birmingham UK

2 Department of Ophthalmology, New Zealand National Eye Centre, The University of Auckland, Auckland, New Zealand

Corresponding author: James S. Wolffsohn, Aston University, School of Life and Health

Sciences, Aston Triangle, Birmingham, B4 7ET, UK j.s.w.wolffsohn@aston.ac.uk

\section{Abstract}

Aim: To evaluate the patient-applied Optrex ${ }^{\mathrm{TM}}$ Dry Eye Blink Test against established clinical criteria for the diagnosis of dry eye disease and to evaluate its benefit in enhancing screening for DED.

Methods: Eighty-seven participants aged $38 \pm 17$ years, (44 female) were screened for dry eye disease using the Tear Film and Ocular Surface Society (TFOS) Dry Eye Workshop II diagnostic criteria. In addition to symptoms screening with the Ocular Surface Disease Index questionnaire ( $\geq 13$ cut-off for DED), a sign of loss of homeostasis of the tear film in the form of a non-invasive tear breakup time (NIBUT) $<10$ s (Oculus Keratograph; $\mathrm{K} 5 \mathrm{~m}$ ), an osmolarity reading $\geq 308 \mathrm{mOsm} / \mathrm{L}$ or an interocular difference in osmolarity of $>8$ (Tearlab), or ocular surface staining ( $>5$ fluorescein corneal spots, $>9$ lissamine green spots or lid wiper staining [ $\geq 2 \mathrm{~mm}$ length $\& \geq 25 \%$ width]) was required to confirm a diagnosis of DED. The self-administered Blink Test, which requires the participant to observe an image on a computer screen and report the length of time (in seconds) that they can refrain from blinking without discomfort, was repeated three times.

Results: Using a cut-off of 10 s, the Blink Test demonstrated sensitivity of $66 \%$, specificity of $88 \%$, and an area under the curve of $0.77(\mathrm{p}<0.001)$, in predicting a diagnosis of DED according to the TFOS DEWS II criteria. The correlation between the Blink Test and NIBUT was $r=0.47, p<0.001)$. When combined with the screening questionnaire, the sensitivity and specificity of the Blink Test increased to $71 \%$ and $90 \%$ respectively.

Conclusion: The Blink Test offers health professionals without advanced instrumentation, as well as patients, themselves, a rapid method of identifying possible DED. 


\section{Introduction}

"Dry eye disease (DED) is a multifactorial disease of the ocular surface characterized by a loss of homeostasis of the tear film, and accompanied by ocular symptoms, in which tear film instability and hyperosmolarity, ocular surface inflammation and damage, and neurosensory abnormalities play etiological roles".[1] The Tear Film and Ocular Surface Society (TFOS) Dry Eye Workshop (DEWS) II provided this updated definition, focusing on the loss of homeostasis in addition to symptoms, as the central pathophysiological concept of dry eye disease (DED). Tear film instability is considered to be a principal factor in the vicious cycle of disease, perpetuating tear film hyperosmolarity and ocular surface inflammation leading to ocular surface damage.[2]

The prevalence of DED has been estimated to be between $5 \%$ and $50 \%$,[3] although the presence of signs and symptoms has been a prerequisite of few studies to date and none have yet been published conforming to the new global consensus on DED diagnosis. Globally, the incidence has been found to be higher in Asian, than in Caucasian, populations. DED prevalence increases approximately linearly with age, with a steeper rise by decade for clinical signs than for the prevalence of symptoms. Sex-related differences become significant only with increasing age.[3]

DED causes both an economic and social burden on those who suffer with this chronic condition.[4] It has been shown that DED can reduce the everyday quality of life of those with the disease.[5] Mild to severe DED has been associated with anxiety and depression.[6-8] In particular, patients suffering from DED secondary to Sjögren's syndrome reportedly have a high prevalence and severity of depression.[9]

The prevalence of ocular symptoms related to DED is difficult to quantify, as symptoms are not spontaneously reported by many patients unless specifically asked. Hence, non-selective use of validated questionnaires such as the Dry Eye Questionnaire 5item [10] or Ocular Surface Disease Index (OSDI)[11] can aid in screening for DED. It's also important, through differential diagnosis, to exclude non-dry eye conditions that can mimic some of the DED signs and symptoms such as ocular allergy, infection and even binocular vision anomalies.[12, 13] Exclusion of such non-dry eye cases, together with a positive symptom score, prompts testing to identify whether there is an accompanying loss of homeostasis, and this is most often undertaken in a specialist clinic due to the time and equipment requirements. Tests recommended by TFOS DEWS II for identifying the loss of homeostasis of the tear film are those that evaluate non-invasive breakup time (with a cut-off time of $<10$ s deemed to indicate DED), 
osmolarity (with a value of $\geq 308 \mathrm{mOsm} / \mathrm{L}$ or a difference of $>8 \mathrm{mOsm} / \mathrm{L}$ between the two eyes), and ocular surface corneal staining ( $>5$ fluorescein spots), conjunctival staining (>9 lissamine green spots) or lid wiper epitheliopathy at the inner eyelid margin (stained zone width with the combined dyes of $\geq 25 \%$ and length $\geq 2 \mathrm{~mm}$ ). [12] If any one of these tests identifies a loss of homeostasis then, in conjunction with the previously confirmed symptomology, a diagnosis of DED is made.

Since pharmacists and general practitioners most often lack access to the technology/dyes for diagnosing dry eye or monitoring treatment effects, availability of a rapid self-assessment test for dry eye could allow non-eyecare practitioners to give better advice to patients and make more appropriate referrals for a full diagnosis or differential diagnosis. Raising awareness of possible dry eye in patients themselves, might also encourage affected individuals to seek appropriate advice and management. The present investigation thus sought to validate a patient-selfassessed test (Optrex ${ }^{\mathrm{TM}}$ Dry Eye Blink Test) and determine the level of certainty with which a diagnosis of DED might be made in the absence of specialist equipment against an established diagnosis, according to TFOS DEWS II. 


\section{Methods}

This study was conducted in accordance with the principles of the Declaration of Helsinki and approved by the institutional ethics committees of Aston University, Birmingham, United Kingdom and the University of Auckland, Auckland, New Zealand. Study participants were recruited from both centres and were enrolled following explanation of the procedures and after providing written informed consent. Eighty-

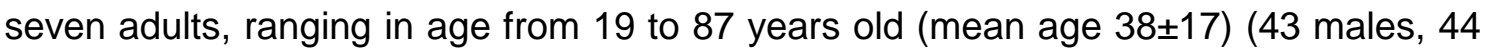
females) were enrolled in the study. All subjects were in good general health and were able to participate in the test sessions without difficulty. Subjects with any active ocular disease or currently using ocular medications were excluded. None of the subjects were contact lens wearers. In particular subjects with a range of DED were recruited to test the discriminatory ability of the Blink Test.

Room conditions were maintained at a temperature of $20.8 \pm 2.1^{\circ} \mathrm{C}$ and relative humidity of $46.1 \pm 9.2 \%$, and subjects spent a minimum of 10 minutes acclimatising to the study room conditions before measurements were captured. The participants were asked to answer the 5 item DEQ questionnaire which scores eye discomfort and eye dryness frequency from 0 (never) to 4 (constantly) and intensity from 0 (never have it) to 5 (very intense) along with watery eye frequency. In addition they scored the 12 item OSDI questionnaire, which scores experience of 3 ocular symptom questions, 6 visual function-related questions and 3 environmental trigger questions during their previous week from 0 (none of the time) to 4 (all of the time). The final score is calculated with the following formula;

$$
\text { OSDI }=\frac{(\text { sum of scores }) \times 25}{(\text { number } \text { of questions answered })}
$$

DED signs were assessed from the right eyes of subjects only in the following order. Non-invasive tear film stability (NIBUT) was assessed objectively with the Keratograph $5 \mathrm{M}$ (Oculus, Wetzlar, Germany) using the infrared light setting, after two non-forceful blinks, from automated detection of disruption of a projected mire pattern reflected from the tear film surface, during a period of non-blinking.[14, 15] An average of three 'average breakup time' measurements was recorded each time.

The mean values of three consecutive Blink Test measurements were obtained (the manufacturer's instructions does not specify repetition). Test instructions were displayed on a 15-inch thin-film-transistor liquid-crystal display digital screen positioned at $\sim 40 \mathrm{~cm}$ from the participant. The patient was requested to make 2 non-forceful 
blinks, before ceasing blinking, and to press a timer button on the screen when discomfort was noted (Figure 1).

Figure 1: The Optrex ${ }^{T M}$ Dry Eye Blink Test (with permission from Reckitt Benckiser)

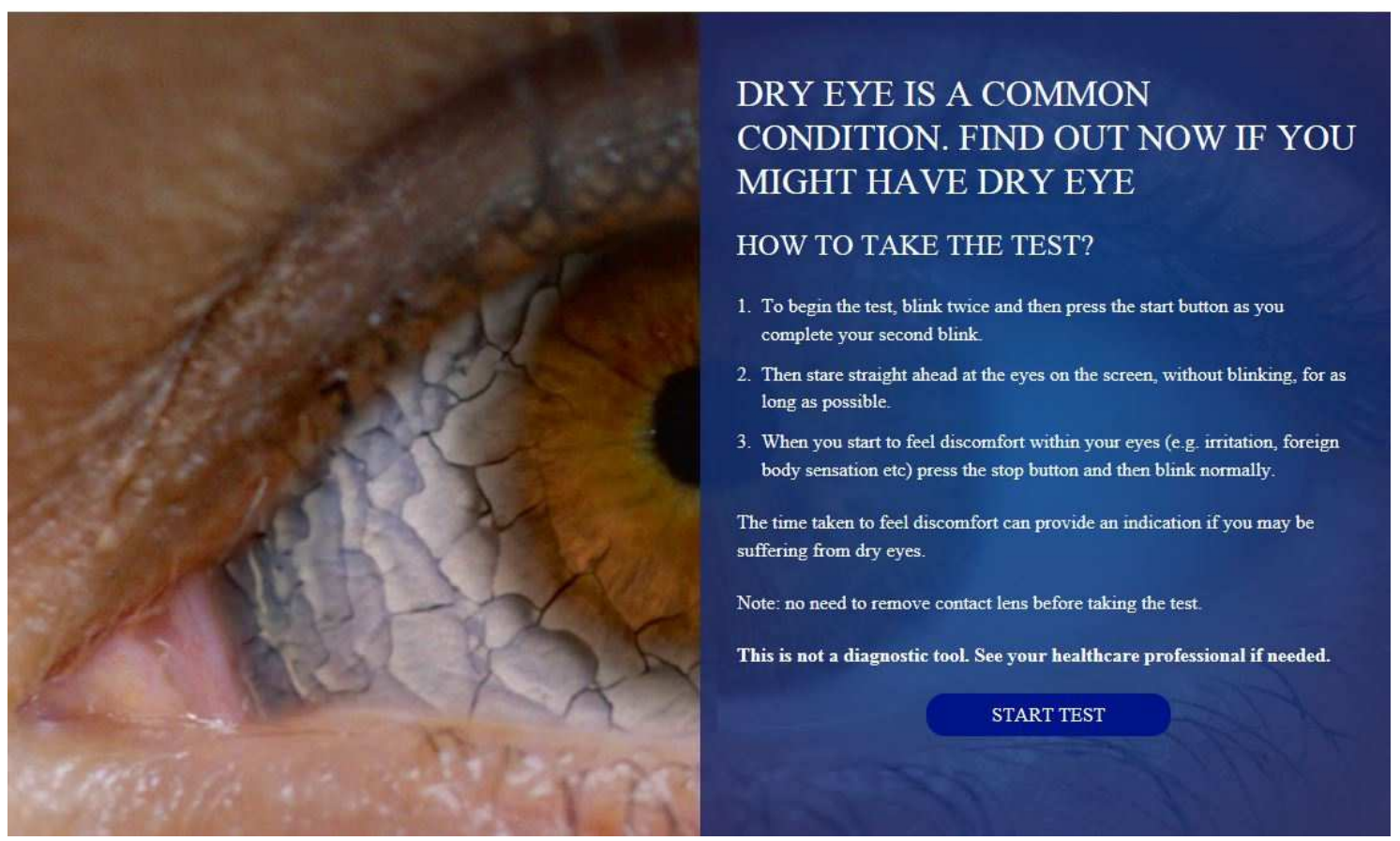

Tear film osmolarity was measured at the inferior tear meniscus with an impedancebased, low volume osmometer (TearLab, San Diego, USA).[16] Daily instrument calibration was performed and stability in temperature ensured in accordance with the manufacturer's instructions.[12] Measures were collected non-invasively from each eye, and the maximum osmolarity and interocular difference recorded.

Conjunctival damage was evaluated with the aid of lissamine green dye (Green Glo, HUB Pharmaceuticals LLC, Rancho Carcamonga, CA, USA). The strip was wet with saline and the lissamine green solution instilled at the temporal canthus, after a period of 5 s to ensure an adequate concentration of lissamine green. Care was taken to avoid conjunctival or lid wiper tissue damage. Grading was conducted between 1 to 4 minutes after instillation (ocular staining score 0: 0-9 dots; 1: 10-32 dots; 2: 33-100 dots; 3 : >100 dots). Corneal damage was evaluated using sodium fluorescein strips. The strip was wet with saline solution and the excess shaken off to minimise instillation volume. Grading (ocular staining score 0: 0 dots; 1: 1-5 dots; 2: 6-30 dots; 3: >30 dots)[17] was conducted between 1 and 3 minutes after instillation.[18] Lid wiper epitheliopathy was observed after corneal and conjunctival grading, and was graded ( 0 : 
length $<2 \mathrm{~mm},<25 \%$ of the lid wiper; 1 : length $2-4 \mathrm{~mm}, 25 \%-<50 \%$ of the lid wiper; 2 : length $5-9 \mathrm{~mm}, 50 \%-<75 \%$ of the lid wiper; 3 : length $>10 \mathrm{~mm}, \geq 75 \%$ of the lid wiper)[19] 3-6 minutes after instillation of a further drop of lissamine green from a new strip by one of three trained researchers.[12]

\section{Data analysis}

Statistical analysis was performed using Graph Pad Prism version 6.02 (California, USA) and IBM SPSS Statistics version 23 (New York, USA). Due to the Blink Test having a maximum duration of $15 \mathrm{~s}$, all NIBUT durations $>15 \mathrm{~s}$ were recorded as $15 \mathrm{~s}$. The distributions of all continuous variables were assessed using the KolmogorovSmirnov normality test, and non-normally distributed measurements (blink test value and non-invasive tear film breakup time) underwent logarithmic transformation before further analysis. The mean blink test and non-invasive tear film breakup time were compared using the paired t-test, variances assessed using the F-test, and BlandAltman analysis then performed to determine the level of agreement between the two measurements. Correlation analysis of ocular measurements with the blink test value was conducted using Spearman's rank correlation coefficient. A receiver operative characteristic (ROC) curve was constructed to assess the diagnostic ability of the Blink Test measurements in discriminating whether or not subjects fulfilled the TFOS DEWS II dry eye diagnostic test battery. The area under the curve (C-statistic) and diagnostic accuracy values at the Youden's optimal diagnostic cut-off were then calculated. All tests were two-tailed and $p<0.05$, considered significant.

A sample size of 85 was calculated to significantly power an association of $r \geq 0.30$ between the Blink Test result, NIBUT and other DED variables. 


\section{Results}

Of the 87 participants enrolled, $62(71 \%)$ subjects fulfilled the TFOS DEWS II diagnostic criteria for DED (Table 1).

Table 1: Demographic, ocular surface, and tear film characteristics of the study participants. Data are presented as mean $\pm S D$, median (IQR), or the number of participants (\% of participants).

\begin{tabular}{lc}
\hline \multicolumn{1}{c}{ Characteristic } & Values \\
\hline Age (years) & $38 \pm 17$ \\
\hline Female sex & $44(51 \%)$ \\
\hline Ethnicity & $38(44 \%)$ \\
\hline European & $40(46 \%)$ \\
\hline East Asian & $4(5 \%)$ \\
\hline South Asian & $5(6 \%)$ \\
\hline Other & $62(71 \%)$ \\
\hline TFOS DEWS II dry eye diagnostic criteria met & $19 \pm 16$ \\
\hline OSDI score & $9 \pm 4$ \\
\hline DEQ-5 score & $305 \pm 14$ \\
\hline Tear film osmolarity (mOsm/L) & $9 \pm 7$ \\
\hline Inter-ocular difference in osmolarity (mOsm/L) & $9.5 \pm 7.3$ \\
\hline Non-invasive tear film breakup time (s) & $9.8 \pm 4.0$ \\
\hline Optrex Blink Test value (s) & $0(0-1)$ \\
\hline Corneal staining score & $0(0-1)$ \\
\hline Conjunctival staining score & $0(0-0)$ \\
\hline Superior lid wiper epitheliopathy grade & $0(0-2)$ \\
\hline Inferior lid wiper epitheliopathy grade &
\end{tabular}

Comparative analysis between the Blink Test and non-invasive tear film stability

The strength of the association between the Blink Test, as a patient-reported 'equivalent' to NIBUT, and NIBUT itself, was evaluated. Following logarithmic transformation, the mean Blink Test and non-invasive tear film stability measurements did not differ significantly $(p=0.15)$. The F-test of variances showed that the Blink Test had a narrower distribution than non-invasive tear film breakup time $(p<0.001)$. A significant positive correlation was observed between the two measurements $\left(r_{s}=0.47\right.$, $\mathrm{p}<0.001)$. Bland-Altman analysis (Figure 2) showed a mean bias $(95 \%$ limits of agreement) of $-0.3( \pm 13.5) \mathrm{s}$. Adjusting the Bland-Altman mean biases to the pretransformed equivalents, the blink test values were on average 1.13 (0.36 to 3.55) times those of non-invasive tear film stability.

Figure 2: Bland-Altman analysis of difference between non-invasive tear breakup time and Blink test. Solid line represent the bias and dotted lines the $95 \%$ limits of agreement. $\mathrm{N}=87$. 


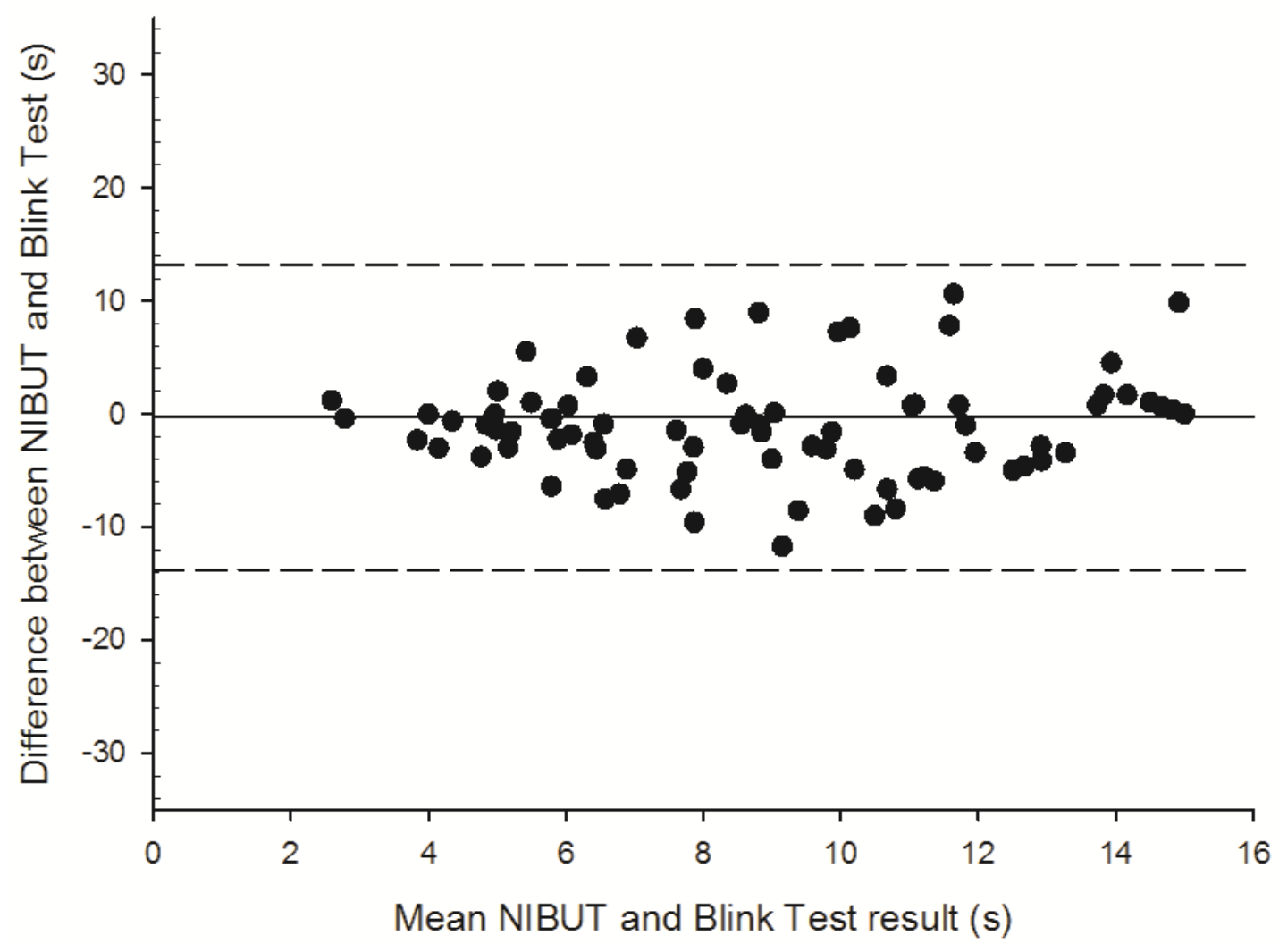

Correlation analysis of DED signs and symptoms with the Blink Test

Correlation analyses of dry eye signs and symptoms with the Blink Test value are presented in Table 2. Significant negative correlations with OSDI, DEQ-5, conjunctival staining, and inferior lid wiper epitheliopathy were observed (all $p<0.05$ ).

Table 2: Correlation analysis of dry eye signs and symptoms with the Blink Test. Data are presented as Spearman's rank correlation coefficients. Asterisks denote statistically significant values $(p<0.05)$.

\begin{tabular}{lcc}
\hline \multirow{2}{*}{ Ocular measurement } & \multicolumn{2}{c}{$\begin{array}{c}\text { Correlation with } \\
\text { Blink Test value }\end{array}$} \\
\cline { 2 - 3 } & Coefficient & $\mathbf{p}$ \\
\hline OSDI Score & -0.290 & $0.006^{*}$ \\
\hline DEQ-5 Score & -0.364 & $0.004^{*}$ \\
\hline Tear film osmolarity (higher value) (mOsm/L) & -0.066 & 0.55 \\
\hline Interocular difference in osmolarity (mOsm/L) & -0.010 & 0.93 \\
\hline Corneal staining score & -0.163 & 0.13 \\
\hline Conjunctival staining score & -0.237 & $0.03^{*}$ \\
\hline Superior lid wiper epitheliopathy grade & 0.018 & 0.87 \\
\hline Inferior lid wiper epitheliopathy grade & -0.251 & $0.02^{*}$ \\
\hline
\end{tabular}




\section{Diagnostic ability of the Blink Test}

The Blink Test was a moderately strong indicator of a DED diagnosis according to TFOS DEWS II (Table 3; Figure 3). The average of three repeats of the Blink Test was more sensitive than a single measure and resulted in a greater area under the receiver operating curve.

Table 3: Diagnostic accuracy indicators of the Blink Test (single measure or average of 3 values) versus the TFOS DEWS II diagnostic criteria or signs of homeostasis.

\begin{tabular}{|c|c|c|c|c|}
\hline \multirow[b]{2}{*}{ Diagnostic accuracy value } & \multicolumn{2}{|c|}{$\begin{array}{c}\text { TFOS DEWS II diagnostic } \\
\text { criteria } \\
\end{array}$} & \multicolumn{2}{|c|}{$\begin{array}{c}\text { TFOS DEWS II signs of } \\
\text { homeostasis }\end{array}$} \\
\hline & $\begin{array}{c}\text { Blink Test } \\
\text { (averaged value) }\end{array}$ & $\begin{array}{c}\text { Blink Test } \\
\text { (single value) }\end{array}$ & $\begin{array}{c}\text { Blink Test } \\
\text { (averaged } \\
\text { value) }\end{array}$ & $\begin{array}{c}\text { Blink Test } \\
\text { (single value) }\end{array}$ \\
\hline C-statistic, 95\% Cl & $\begin{array}{l}0.77(0.68 \text { to } \\
0.87)\end{array}$ & $\begin{array}{l}0.70(0.59 \text { to } \\
0.82)\end{array}$ & $\begin{array}{l}0.81(0.71 \text { to } \\
0.91)\end{array}$ & $\begin{array}{c}0.70(0.55 \text { to } \\
0.85)\end{array}$ \\
\hline C-statistic p-value & $<0.001^{*}$ & $0.003^{*}$ & $0.001^{*}$ & $0.04^{*}$ \\
\hline $\begin{array}{l}\text { Youden's optimal diagnostic } \\
\text { cut-off }\end{array}$ & $\leq 10$ & $<8$ & $\leq 11$ & $\leq 8$ \\
\hline Sensitivity, 95\% Cl & $\begin{array}{c}66 \%(53 \% \text { to } \\
78 \%)\end{array}$ & $\begin{array}{c}53 \%(40 \% \text { to } \\
66 \%)\end{array}$ & $\begin{array}{c}71 \%(60 \% \text { to } \\
81 \%)\end{array}$ & $\begin{array}{c}52 \%(40 \% \text { to } \\
63 \%)\end{array}$ \\
\hline Specificity, 95\% Cl & $\begin{array}{l}88 \%(69 \% \text { to } \\
97 \%)\end{array}$ & $\begin{array}{c}88 \%(69 \% \text { to } \\
98 \%)\end{array}$ & $\begin{array}{c}90 \%(56 \% \text { to } \\
100 \%)\end{array}$ & $\begin{array}{c}90 \%(56 \% \text { to } \\
100 \%)\end{array}$ \\
\hline $\begin{array}{l}\text { Positive predictive ability, } 95 \% \\
\mathrm{Cl}\end{array}$ & $\begin{array}{c}93 \%(82 \% \text { to } \\
98 \%)\end{array}$ & $\begin{array}{c}92 \%(79 \% \text { to } \\
97 \%)\end{array}$ & $\begin{array}{c}98 \%(90 \% \text { to } \\
100 \%)\end{array}$ & $\begin{array}{c}98 \%(86 \% \text { to } \\
100 \%)\end{array}$ \\
\hline $\begin{array}{l}\text { Negative predictive ability, } \\
95 \% \mathrm{Cl}\end{array}$ & $\begin{array}{c}51 \%(42 \% \text { to } \\
60 \%)\end{array}$ & $\begin{array}{c}43 \%(36 \% \text { to } \\
51 \%)\end{array}$ & $\begin{array}{c}29 \%(21 \% \text { to } \\
38 \%)\end{array}$ & $\begin{array}{c}20 \%(15 \% \text { to } \\
25 \%)\end{array}$ \\
\hline $\begin{array}{l}\text { Positive likelihood ratio, 95\% } \\
\mathrm{Cl}\end{array}$ & $\begin{array}{c}5.51(1.88 \text { to } \\
16.17)\end{array}$ & $\begin{array}{c}4.44(1.50 \text { to } \\
13.15)\end{array}$ & $\begin{array}{l}7.14(1.11 \text { to } \\
46.10)\end{array}$ & $\begin{array}{l}5.19(0.80 \text { to } \\
33.77)\end{array}$ \\
\hline $\begin{array}{l}\text { Negative likelihood ratio, 95\% } \\
\mathrm{Cl}\end{array}$ & $\begin{array}{c}0.38(0.26 \text { to } \\
0.56)\end{array}$ & $\begin{array}{l}0.53(0.39 \text { to } \\
0.72)\end{array}$ & $\begin{array}{l}0.32(0.21 \text { to } \\
0.48)\end{array}$ & $\begin{array}{l}0.53(0.39 \text { to } \\
0.73)\end{array}$ \\
\hline
\end{tabular}


Figure 3: Receiver operative characteristic curves assessing the diagnostic ability of the Blink Test in discriminating whether subjects fulfilled the (a) TFOS DEWS II dry eye diagnostic test battery or (b) homeostasis marker signs.

\section{a) Dry eye diagnostic test battery}

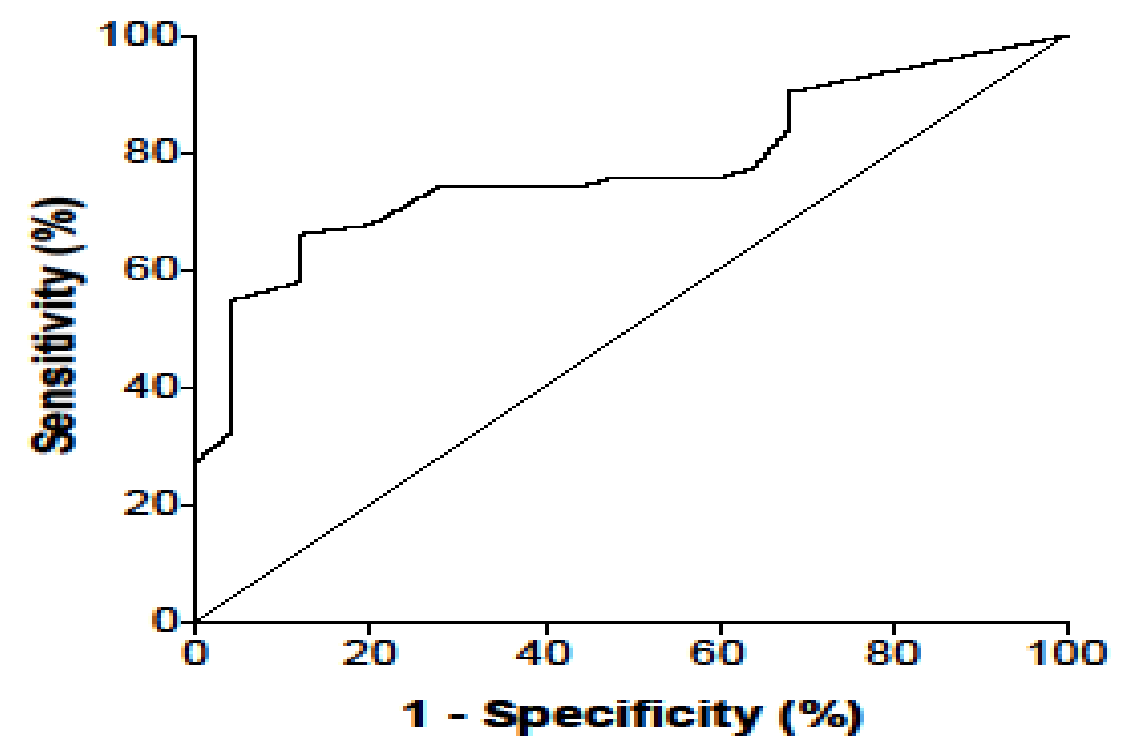

\section{b) Homeostasis markers}

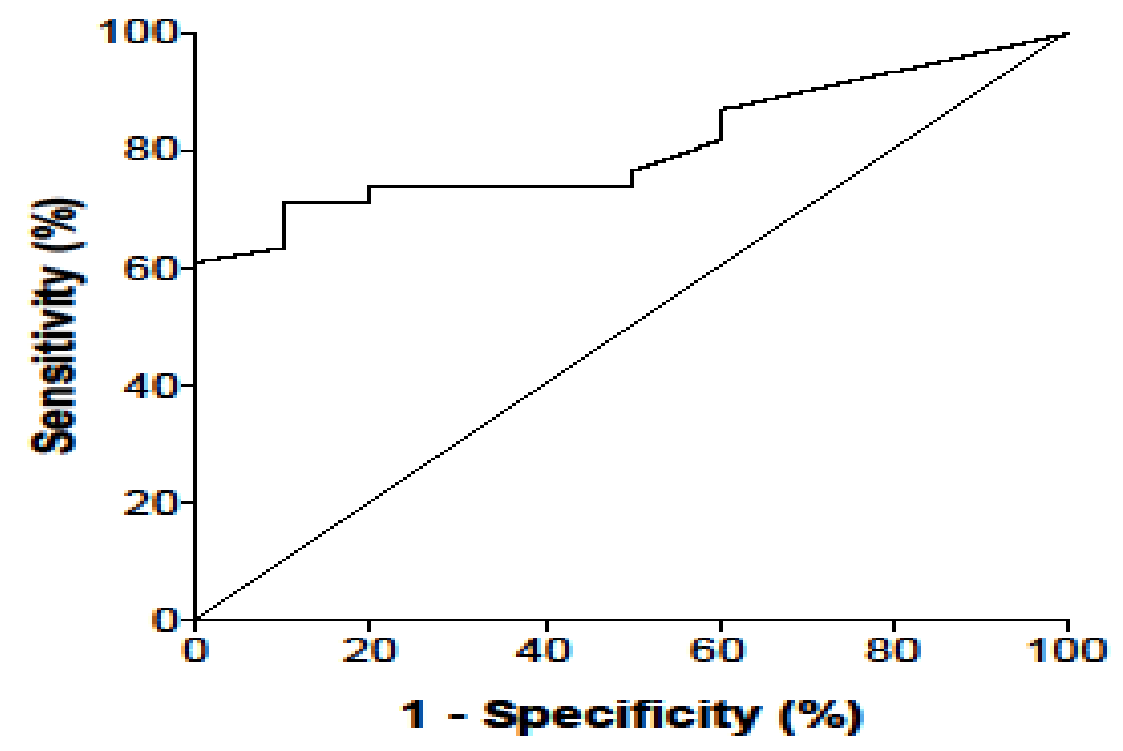




\section{Discussion}

The Optrex ${ }^{\mathrm{TM}}$ Dry Eye Blink Test is advertised as a rapid online test to indicate whether an individual might have dry eye. Like the clinical tear breakup test, it reflects the stability of the tears, however, in this test the time taken for the eyes to sense ocular discomfort is the measure for dry eye rather than the clinical assessment of when the tear film first 'breaks'. However, the diagnostic potential of the self-reported Blink Test approach has not been investigated previously. In order to test the validity of this approach, the current study examined how well the Blink Test performed against the TFOS DEWS II diagnostic criteria for dry eye disease.

The results showed that the mean blink test and NIBUT results were not significantly different. The Blink Test has a narrower distribution than NIBUT, even when NIBUT is capped at $15 \mathrm{~s}$, hence in its current form, the disagreement beyond this point will increase. Bland-Altman analysis showed fair levels of clinical agreement between the two tests, however the limits of agreement were moderately wide. As expected, there was also a positive correlation between the tests, with the blink time increasing as NIBUT increased. Tear film breakup is associated with increasing ocular discomfort and this is well documented in the literature.[20, 21] It is thought that tear breakup during the blink-interval results in a transient, localised, increase in tear osmolarity, which in turn stimulates the corneal nociceptors, driving the blink reflex to replenish the tear film.[21] If the breakup of tears on the ocular surface led directly to a rapid loss of comfort, it would be expected that the correlation between NIBUT and the Blink Test would have been greater than moderate. Hence the results suggest that other individual factors, such as the patient's tolerance to pain, the area of breakup and osmolarity levels, affect when patients report discomfort. A more frequent blink rate has been associated with a shorter NIBUT and this may be a compensatory mechanism to mitigate the effects of reduced tear stability.[22] It is known that blink rate diminishes during tasks involving concentration, which may explain why ocular discomfort is commonly experienced by computer users.[23] The blink test, being an online tool, is a relevant tool for such individuals, amongst whom evaporative dry eye is a rising issue. Although it does not simulate a truly natural state, it may reflect the typical stresses on the tear film that occur during the day. The Blink Test also demonstrated a significant negative correlation with conjunctival staining levels such that longer Blink Test time were associated with higher symptomology scores and a lower level of ocular surface 
staining. While other ocular measures of dry eye showed similarly negative trends with higher Blink Test scores, these were not statistically significant.

Poor correlation is not infrequently reported between objective clinical test outcomes and symptom severity.[24] This may be due partly to a degree of compensation by blinking more frequently which would serve to reduce the time the tear film needs to maintain its interblink homeostasis. The instantaneous and quantitative Blink Test has the potential to be a more reliable indicator of dry eye risk, by overcoming issues associated with inaccurate patient recall of discomfort symptoms over the previous month, as posed by typical dry eye questionnaires. According to the Blink Test instructions, contact lenses do not need to be removed before taking the test, widening its applicability to a broader patient group. The validity of the test in this demographic was not addressed in the present study, but requires independent evaluation. Contact lenses may act as a partial barrier to corneal sensation,[20] and therefore their wear during the test might be expected to overestimate the Blink Test time (giving a false negative result).

Test sensitivity is a measure of how well a test correctly identifies those with disease, whereas specificity evaluates how well a test can correctly identify those without disease. A trade-off typically exists between the two with the precise values for each influenced by the selected cut-off value for a positive diagnosis. Maximal sensitivity and specificity for the Blink Test was achieved with a cut-off of $\leq 10 \mathrm{~s}$. This offered a sensitivity and specificity of $66 \%$ and $88 \%$, respectively. The ROC curve (Figure 3) shows this graphically, with the area under the curve demonstrating high diagnostic ability $(A \cup C=0.77, P<0.001)$. Interestingly the optimal cut-off for the Blink Test aligned closely with that reported by TFOS DEWS II, for NIBUT.[12]

As questionnaires are rapid and simple to administer outside a clinical setting, the sensitivity and specificity of the Blink Test as a surrogate for detecting a loss of homeostasis as classified by TFOS DEWS II,[12] was also assessed. Screening out non-symptomatic individuals first, demonstrated improved discrimination $(71 \%$ sensitivity, $90 \%$ specificity and an AUC of 0.81 ), compared with the Blink Test alone. It was further examined whether a single assessment of the Blink test would be adequate to save testing time, however, it was clearly demonstrated that, like NIBUT measurement in clinical practice, an average of three repeats of the Blink Test improves diagnostic sensitivity and therefore should be recommended. 
All procedures on a participant were carried out by a single examiner, therefore investigator-masking was not possible. However, all were objective or participant completed so operator bias in the results is unlikely. Ocular surface grading was completed subsequent to the Blink Test and without reference to the other clinical findings, and so was effectively masked. As the Blink Test is designed for completion in non-clinical settings, the working distance used was only approximately $40 \mathrm{~cm}$ and the gaze angle will have differed depending on patient height and sitting stature. Screen illumination and screen size could also affect the blink rate and induce reflex tearing so are other factors that could affect the result. However, despite this, the Blink Test was demonstrated to be a useful indicator of the presence of DED that can be easily applied in non-specialist eye-care settings to aid in the identification and management monitoring of this chronic, debilitating disease, with a view to improving patient outcomes.

\section{Declaration of Interests}

No funding was received to support this study. None of the authors have any proprietary interests relating to this research. 


\section{References}

1. Craig, J.P., et al., TFOS DEWS II Definition and Classification Report. Ocul Surf, 2017. 15(3): p. 276-283.

2. Belmonte, C., et al., TFOS DEWS II pain and sensation report. Ocul Surf, 2017. 15(3): p. 404-437.

3. $\quad$ Stapleton, F., et al., TFOS DEWS II Epidemiology Report. Ocul Surf, 2017. 15(3): p. 334365.

4. McDonald, M., et al., Economic and Humanistic Burden of Dry Eye Disease in Europe, North America, and Asia: A Systematic Literature Review. Ocul Surf, 2016. 14(2): p. 144-67.

5. Li, M., et al., Assessment of vision-related quality of life in dry eye patients. Invest Ophthalmol Vis Sci, 2012. 53(9): p. 5722-7.

6. Li, M., et al., Anxiety and depression in patients with dry eye syndrome. Curr Eye Res, 2011. 36(1): p. 1-7.

7. Kim, K.W., et al., Association between depression and dry eye disease in an elderly population. Invest Ophthalmol Vis Sci, 2011. 52(11): p. 7954-8.

8. van der Vaart, R., et al., The association between dry eye disease and depression and anxiety in a large population-based study. Am J Ophthalmol, 2015. 159(3): p. 470-4.

9. Wan, K.H., L.J. Chen, and A.L. Young, Depression and anxiety in dry eye disease: a systematic review and meta-analysis. Eye (Lond), 2016. 30(12): p. 1558-1567.

10. Chalmers, R.L., C.G. Begley, and B. Caffery, Validation of the 5-Item Dry Eye Questionnaire (DEQ-5): Discrimination across self-assessed severity and aqueous tear deficient dry eye diagnoses. Cont Lens Anterior Eye, 2010. 33(2): p. 55-60.

11. Dougherty, B.E., J.J. Nichols, and K.K. Nichols, Rasch analysis of the Ocular Surface Disease Index (OSDI). Invest Ophthalmol Vis Sci, 2011. 52(12): p. 8630-5.

12. Wolffsohn, J.S., et al., TFOS DEWS II Diagnostic Methodology report. Ocul Surf, 2017. 15(3): p. 539-574.

13. Rueff, E.M., P.E. King-Smith, and M.D. Bailey, Can Binocular Vision Disorders Contribute to Contact Lens Discomfort? Optom Vis Sci, 2015. 92(9): p. e214-21.

14. Best, N., L. Drury, and J.S. Wolffsohn, Clinical evaluation of the Oculus Keratograph. Cont Lens Anterior Eye, 2012. 35(4): p. 171-4.

15. Abdelfattah, N.S., et al., Noninvasive Imaging of Tear Film Dynamics in Eyes With Ocular Surface Disease. Cornea, 2015. 34 Suppl 10: p. S48-52.

16. Gokhale, M., U. Stahl, and I. Jalbert, In situ osmometry: validation and effect of sample collection technique. Optom Vis Sci, 2013. 90(4): p. 359-65.

17. Whitcher, J.P., et al., A simplified quantitative method for assessing keratoconjunctivitis sicca from the Sjogren's Syndrome International Registry. Am J Ophthalmol, 2010. 149(3): p. 405-15.

18. Peterson, R.C., J.S. Wolffsohn, and C.W. Fowler, Optimization of anterior eye fluorescein viewing. Am J Ophthalmol, 2006. 142(4): p. 572-5.

19. Korb, D.R., et al., Lid wiper epitheliopathy and dry eye symptoms. Eye Contact Lens, 2005. 31(1): p. 2-8.

20. Zhang, J., et al., $A$ link between tear breakup and symptoms of ocular irritation. Ocul Surf, 2017. 15(4): p. 696-703.

21. Varikooty, J. and T.L. Simpson, The interblink interval I: the relationship between sensation intensity and tear film disruption. Invest Ophthalmol Vis Sci, 2009. 50(3): p. 1087-92.

22. Nosch, D.S., et al., Relationship between Corneal Sensation, Blinking, and Tear Film Quality. Optom Vis Sci, 2016. 93(5): p. 471-81.

23. Portello, J.K., M. Rosenfield, and C.A. Chu, Blink rate, incomplete blinks and computer vision syndrome. Optom Vis Sci, 2013. 90(5): p. 482-7. 
24. Nichols, K.K., J.J. Nichols, and G.L. Mitchell, The lack of association between signs and symptoms in patients with dry eye disease. Cornea, 2004. 23(8): p. 762-70. 